\title{
Uma investigação empírica do desempenho da amostragem compressiva em codificação de imagens
}

\author{
Adriana Schulz ${ }^{1}$, Luiz Velho ${ }^{1}$, Eduardo A. B. da Silva ${ }^{2}$ \\ ${ }^{1}$ IMPA - Instituto de Matemática Pura e Aplicada, Brasil \\ ${ }^{2}$ PEE/COPPE/DEL/Poli, Univ. Fed. do Rio de Janeiro, Brasil \\ e-mails: aschulz@impa.br,lvelho@impa.br,eduardo@lps.ufrj.br
}

\begin{abstract}
Resumo-Amostragem compressiva (Compressive SensingCS) é um novo paradigma para aquisição e compressão de dados que tem atraído o interesse da comunidade de processamento de sinais. No contexto de compressão de imagens, é relevante estimar o número de bits necessários para atingir uma qualidade específica. Embora tenham sido publicados recentemente diversos resultados teóricos referentes ao desempenho em taxa-distorção de CS, ainda não existem disponíveis muitos resultados práticos em compressão de imagens. $O$ objetivo deste artigo é avaliar empiricamente o desempenho em taxa-distorção de aplicações de CS à compressão de imagens. São analisadas questões como o algoritmo de minimização utilizado e a transformada empregada, assim como o compromisso entre o número de medidas e o erro de quantização. A partir dos resultados experimentais obtidos destacam-se as potencialidades e as limitações de CS quando comparada aos métodos tradicionais de compressão de imagem.
\end{abstract}

Palavras-Chave-Compressive Sensing (CS), Análise TaxaDistorção, Quantização, Compressão de Imagens.

\section{INTRODUÇÃO}

Imagens comuns, assim como a maioria dos sinais naturais e gerados pelo ser humano, são compressíveis e, portanto, podem ser bem representados em um domínio no qual o sinal é esparso. Técnicas padrão de aquisição de imagens seguem o paradigma amostragem seguido de compressão. Isto envolve amostrar a uma taxa alta e, em seguida, descartar a maior parte da informação adquirida a partir de um esquema de compressão que explora a representação esparsa.

Neste contexto, CS surge como um novo paradigma para aquisição de dados. CS apresenta um algoritmo estável e robusto que permite, em diversos casos, amostrar em taxas muito menores que o limite de Nyquist e, paralelamente, recuperar o sinal com pouca distorção [1], [2]. A reconstrução a partir das medidas é obtida por meio de técnicas de otimização convexa (e.g., minimização da norma $l_{1}$ ).

Para comprimir uma informação medida, é necessário fazer uso de esquemas de quantização que somam distorções aos dados adquiridos. Assim, uma contribuição relevante à teoria de CS consiste em verificar seu desempenho diante de erros de quantização e avaliar seu comportamento em termos de taxa-distorção.

Resultados teóricos foram estabelecidos garantindo a estabilidade de CS a erros de quantização. Em [3], a codificação CS de sinais aproximadamente esparsos com medidas quantizadas é estudada e se demonstra que sua eficiência se aproxima da do codificador ótimo a menos de um fator logarítmico.
Trabalhos relacionados consideram sinais esparsos e avaliam CS quando são acrescentados erros de quantização. Em [4], os resultados de [3] são estendidos ao cenário no qual esparsidade estrita é garantida e ineficiências com relação a taxa e desempenho são verificadas, sugerindo modificações no método de quantização uniforme e no algoritmo de reconstrução. Em [5], ambas as modificações são exploradas e simulações computacionais extensivas são feitas confirmando suas vantagens.

A função taxa-distorção é utilizada em [6] para comparar CS ao esquema de compressão ideal (onde um oráculo informa o padrão de esparsidade) e a perda em desempenho é avaliada como sendo relativamente pequena. Em [7], um limite inferior para o número de medidas necessárias para reconstruir o sinal é definida como uma função da SNR das medições e da função taxa-distorção.

No entanto, questões fundamentais relativas ao desempenho de aplicações práticas ainda permanecem sem resposta [8].

Este trabalho visa ajudar a responder algumas destas questões a partir de um ponto de vista empírico. Apesar de terem sido sugeridas inúmeras aplicações para a teoria, nosso estudo será centrado no contexto de aquisição e compressão de imagens.

É importante ressaltar que não pertence ao escopo deste trabalho o aperfeiçoamento de resultados teóricos ou a análise das limitações da técnica em cenários idealizados. $\mathrm{O}$ objetivo é avaliar a aplicações de CS à aquisição de imagens através de uma análise empírica.

\section{Conceitos BÁsicos E NotAÇÃo DE CS}

Seja $x \in \mathbb{C}^{N \times 1}$ a representação vetorial de uma imagem $\mathrm{e}$ $\Psi \in \mathbb{C}^{N \times N}$ uma transformação unitária (e.g., a transformada do cosseno discreta (discrete cosine transform - DCT)) que torna $x$ esparso, i.e., $\Psi x=s$, onde $s$ possui apenas $S$ coeficientes não-nulos.

O objetivo de CS é construir um esquema de aquisição que capture a imagem já em sua forma comprimida, i.e., que tome apenas $M \ll N$ medidas e consiga reconstruir o sinal. Se fosse conhecida a priori a posição dos coeficientes mais significativos no domínio $\Psi$ (como em um esquema linear de compressão), seria possível simplesmente medir seus valores e desconsiderar a investigação de outras informações.

Entretanto, aproximações lineares geralmente apresentam desempenhos que estão longe do ótimo. Assim, apesar de $x$ 
ser esparso em algum domínio, a distribuição os coeficientes mais significativos não é conhecida. Além disso, é desejável obter uma solução não-adaptativa para o problema de forma a possibilitar o uso do mesmo procedimento de captura para qualquer sinal.

Neste contexto, substitui-se amostragens pontuais por medidas lineares mais genéricas dos sinais. Cada medida, $y_{m}$ do sistema de aquisição é o produto interno do sinal $x$ com uma função de teste $\phi_{m}$. Assim, tomar poucas medidas equivale a multiplicar $x$ por uma matriz gorda ${ }^{1} \Phi_{\Omega} \in \mathbb{C}^{M \times N}$, onde cada linha corresponde a uma função de medida $\phi_{m}$.

$$
y=\Phi_{\Omega} x \Longleftrightarrow y=\Theta_{\Omega} s, \text { onde } \Theta_{\Omega}=\Phi_{\Omega} \cdot \Psi^{*}
$$

O problema de reconstruir $x$ a partir de $y$ é mal condicionado. No entanto, nem todas as soluções satisfazem a propriedade de esparsidade de $s$ e, portanto, uma escolha simples consistiria em procurar, entre todas as soluções possíveis, aquela que torna $s$ esparso.

Como esta busca é combinatória e NP-difícil, utiliza-se o fato de sinais esparsos possuírem normas $l_{1}$ relativamente pequenas e recupera-se o sinal original a partir da equação

$$
\min _{s}\|s\|_{l_{1}} \text { sujeito a } \quad \Phi_{\Omega} \cdot \Psi^{*} s=y .
$$

Este é um problema de otimização convexa que é computacionalmente viável.

\section{A. Incoerência}

Para que este algoritmo de aquisição seja eficiente, é necessário impor algumas características à matriz de aquisição $\Phi_{\Omega}$. Se as amostras fossem tomadas no domínio onde o sinal é esparso (i.e., $\Phi_{\Omega}$ é uma restrição a $M$ linhas de $\Phi=\Psi$ ), um grande número de coeficientes nulos seriam adquiridos e não haveria informação suficiente para reconstruir o sinal. No entanto, se os dois domínios ( $\Phi$ e $\Psi$ ) são incoerentes, uma série de combinações aleatórias das entradas é adquirida. Assim, a cada medição, aprende-se algo novo sobre o sinal esparso. Defini-se coerência da seguinte forma.

Definição 1 (Coerência entre $\Psi$ and $\Phi[9])$ :

$$
\mu(\Phi, \Psi)=\sqrt{N} \max _{i, j}\left|\left\langle\phi_{i}, \psi_{j}\right\rangle\right|, \quad\left\|\phi_{i}\right\|_{l_{2}}\left\|\psi_{i}\right\|_{l_{2}}=1
$$

$\mathrm{O}$ seguinte teorema especifica condições que garantem a eficiência do método

Teorema 1 ([10]): Se

$$
M \geq C_{0} \cdot S \cdot \mu^{2}(\Phi, \Psi) \cdot \log (N)
$$

para uma constante numérica $C_{0}$ (razoavelmente pequena). A solução da Equação 1 reconstrói o sinal original com altíssima probabilidade.

\section{B. Robustez}

De forma geral, sinais naturais não são esparsos, mas são aproximadamente esparsos ou possuem um decaimento exponencial. Além disso, medições não são perfeitas e, quase sempre, têm algum ruído adicionado (e.g., erros devido à

\footnotetext{
${ }^{1}$ Usamos o termo gorda para fazer referência a matrizes onde o número de colunas excede o número de linhas.
}

quantização). Mesmo nestes casos, a teoria de CS afirma ser possível recuperar $x$ a partir de $y$ com altíssima probabilidade se $\Theta_{\Omega}$ satisfaz a Propriedade de Isometria Restrita (Restricted Isometry Property - RIP) [11].

Definição 2 (Constante de Isometria Restrita [11]): Para cada inteiro $S=1,2, \ldots, N$ define-se a constante de isometria $S$-restrita $\delta_{S}$ de uma matriz $\Theta_{\Omega}$ como o menor número tal que

$$
\left(1-\delta_{S}\right)\|s\|_{l_{2}}^{2} \leq\left\|\Theta_{\Omega} s\right\|_{l_{2}}^{2} \leq\left(1+\delta_{S}\right)\|s\|_{l_{2}}^{2}
$$

para todos vetores $S$-esparsos.

A isometria restrita é uma propriedade da matriz de medições $\Theta_{\Omega}$ que se refere à existência e limitação de $\delta_{S}$. A RIP é uma extensão do conceito de incoerência e estabelece uma condição que, se obedecida por $\Theta_{\Omega}$, garante a recuperação de sinais não esparsos e com erros nas medições. Note que a constante $\delta_{S}$ é intrínseca à estrutura de $\Theta_{\Omega}$ e, portanto, ao definir restrições para seu tamanho, é possível quantificar a eficiência da matriz de aquisição.

A razão do nome RIP é simples: a energia do sinal restrito ao conjunto $\Omega$ é proporcional ao tamanho de $\Omega$. No entanto, alguns autores descrevem esta propriedade como um Princípio Uniforme da Incerteza (Uniform Uncertainty Principle - UUP) porque ela garante que o sinal não pode estar simultaneamente concentrado nos domínios de esparsidade e de medições.

Seja $s$ um sinal apenas aproximadamente esparso e $s_{S}$ a melhor aproximação $S$-esparsa de $s$, i.e, o resultado obtido quando força-se os $N-S$ menores coeficientes de $s$ a serem zero. Considere também que as medidas $y \in \mathbb{C}^{M \times 1}$ estão corrompidas pela adição de um ruído, i.e., $y=\Phi x+\eta$, onde $\|\eta\|_{l_{2}} \leq \epsilon_{q}$.

Teorema 2 ([12]): Se $\delta_{2 S}<\sqrt{2}-1$, a solução $\hat{s}$ de

$$
\min _{s}\|s\|_{l_{1}} \text { sujeito a }\left\|y-\Theta_{\Omega} s\right\|_{l_{2}} \leq \epsilon_{q} \text {. }
$$

obedece

$$
\left\|y-\Theta_{\Omega} s\right\|_{l_{2}} \leq C \cdot(\epsilon_{q}+\underbrace{S^{-1 / 2}\left\|x_{S}-x\right\|_{l_{1}}}_{\epsilon_{s}}),
$$

onde $\mathrm{C}$ é relativamente pequeno.

É importante observar que o erro de reconstrução é limitado pela superposição dos erros de medições (quantização) e erros decorrentes do sinal não ser estritamente esparso. Disso resulta que a distorção do sinal reconstruído é da ordem do maior valor entre os erros de medição e esparsidade. Para maiores detalhes veja [13].

Um aspecto importante para o uso da técnica na prática é a facilidade de construir matrizes com esta propriedade. Demonstra-se [13] que a RIP pode ser assumida se as linhas de $\Theta_{\Omega}$ pertencerem a certos conjuntos aleatórios e $M$ for da ordem de $S \log (N / M)$.

\section{SETUP EXPERIMENTAL}

Foram consideradas quatro imagens de teste de tamanho $N=256^{2}=65536$, que diferem entre si em termos de esparsidade e de distribuição dos coeficientes de alta energia no domínio da freqüência (veja Figura 1). Como Phantom 
é constante por partes e Lena é suave, a energia é majoritariamente concentrada nos coeficientes de baixa freqüência. Por outro lado, como Text é uma imagem com variações de intensidade abruptas, sua energia está espalhada ao longo de praticamente toda a base DCT. Camera man, por sua vez, tem espalhamento de energia intermediário, apresentando altas intensidades em algumas diagonais do domínio DCT que correspondem às linhas nítidas da imagem.

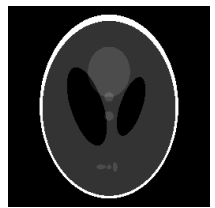

(a) Phantom

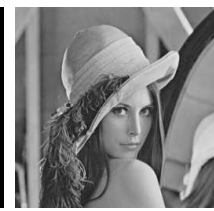

(b) Lena

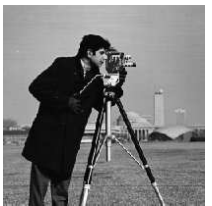

(c) Camera man

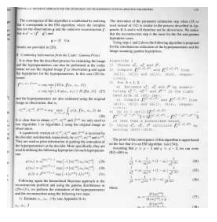

(d) Text
Fig. 1. Imagens de teste.

Como as imagens estão armazenadas no computador como uma matriz de pixels, a aquisição é simulada através de medidas que envolvem combinações lineares destes pixels.

Medições são feitas selecionando-se aleatoriamente $M$ formas de onda de uma transformada Noiselet $N \times N$. Tais medidas foram escolhidas porque são altamente incoerentes com os domínios considerados esparsos e a RIP tende a ser preservada para valores razoáveis de $M$. Além disso, a matriz gerada pela transformação é ortogonal e auto-adjunta, sendo, portanto, de fácil manipulação. Abaixo, encontra-se uma ilustração da matriz de medidas $\Phi$ para $N=4$.

$$
\Phi=\frac{1}{2} \cdot\left[\begin{array}{rrrr}
1 & -1 & 1 & 1 \\
-1 & 1 & 1 & 1 \\
1 & 1 & -1 & 1 \\
1 & 1 & 1 & -1
\end{array}\right]
$$
das:

As seguintes estratégias de reconstrução foram considera-

- DCT- $l_{1}-\mathbf{N}$ Minimização da norma $l_{1}$ da DCT da imagem;

- B_DCT- $l_{1}-\mathbf{N}$ Minimização da norma $l_{1}$ da DCT em blocos da imagem;

- DWT- $l_{1}-\mathbf{N}$ Minimização da norma $l_{1}$ da trasformada Wavelet discreta (discrete Wavelet transform - DWT);

- SVD- $l_{1}$-N Minimização da norma $l_{1}$ da decomposição em valores singulares (singular value decomposition - SVD);

- TV-N Minimização da norma de variação total (total variation - TV) da imagem.

As reconstruções de DCT- $l_{1}-\mathrm{N}$, B_DCT- $l_{1}-\mathrm{N}$, DWT- $l_{1}-\mathrm{N}$ e SVD- $l_{1}-\mathrm{N}$ são baseadas na equação

$$
\hat{s}=\min _{s}\|s\|_{l_{1}} \quad \text { sujeito a } \quad\left\|y-\Phi_{\Omega} \Psi^{*} s\right\|_{l_{2}} \leq \epsilon,
$$

onde $\Phi_{\Omega}$ é a matriz de Noiselet $\Phi$ restrita a $M=|\Omega|$ linhas aleatoriamente selecionadas e, $\Psi$, a matriz que transforma o sinal em sua representação esparsa (DCT, DCT em blocos, DWT e SVD).

A eficiência de cada estratégia está relacionada à esparsidade da imagem no domínio considerado. As transformadas DCT e Wavelets foram escolhidas por sua ampla utilização em padrões de compressão. Considera-se a transformada DCT da imagem completa e da imagem particionada em blocos de tamanho $8 \times 8$ (este valor foi selecionado por ser o utilizado no padrão JPEG). Como a maioria dos teoremas publicados referem-se a bases ortogonias, optou-se por utilizar uma base de Wavelets deste gênero (Coiflet de 2 "vanishing moments") em detrimento de uma base biortogonal, que seria mais eficiente.

Como a esparsidade exerce um papel importante na determinação da eficiência de CS, comparamos seus resultados com os obtidos considerando $\Psi$ a SVD, que determina uma representação altamente esparsa. Esta técnica exige o conhecimento da base de SVD, que é calculada a partir da imagem inteira (não disponível em CS) e exige uma alta taxa de transmissão de dados (que não é levada em consideração). Entretanto, os resultados obtidos por este método são utilizados com limites superiores que, apesar de folgados, permitem discussões interessantes a respeito das limitações de performance.

Em muitas publicações recentes [1], [14], pesquisadores de CS usaram a norma TV, que pode ser interpretada como a norma $l_{1}$ do gradiente (apropriadamente discretizado). A minimização da norma TV favorece certa suavidade, que é freqüentemente encontrada em imagens naturais, sem penalizar características descontinuas. Assim, ela apresenta bons resultados quando aplicada à compressão de imagens. Na estratégia TV-N, as imagens são reconstruídas a partir da solução do seguinte problema de otimização convexa:

$$
\hat{x}=\min _{x}\|x\|_{T V} \quad \text { sujeito a } \quad\left\|y-\Phi_{\Omega} x\right\|_{l_{2}} \leq \epsilon .
$$

\section{A. Implementação}

Os experimentos foram implementados em MATLAB e a toolbox $l_{1}$-Magic [15] foi utilizada para solucionar os problemas de otimização que recuperam as imagens amostradas.

A base de Wavelet foi gerada com o pacote WAVELAB [16] e, a base de Noiselet, usando um algoritmo disponibilizado por Romberg [14].

O parâmetro $\epsilon$ das equações de reconstrução deve ser proporcional a uma limitação da contribuição do ruído e foi selecionado a partir de uma série de experimentos. Calculando a relação sinal ruído de pico (peak signal to noise ratio PSNR) para vários valores de $\epsilon$, é observado um valor ótimo, abaixo do qual a melhor solução está fora do conjunto convexo limitado pelas restrições e acima do qual a solução é menos exata.

\section{Resultados PRELIMINARES}

Antes de avaliar o efeito de quantização sobre CS, é interessante observar como a técnica se comporta no caso ideal (sinais estritamente esparsos e sem erros de medições). Para isto, forçamos esparsidade na imagem Lena, deixando apenas os $S=3.5 k, 6 k, 10 k$ e $14 k$ maiores coeficientes e zerando os demais. A Figura 2 mostra que existe um limiar a partir do qual CS passa a operar eficientemente e que este está altamente relacionado ao valor de $S$ (conforme a Equação 2).

Também é interessante notar que o método é não-adaptativo. Aplicando a estratégia DCT- $l_{1}-\mathrm{N}$ a versões $10 k$-esparsas das quatro imagens de teste e comparando os resultados com 


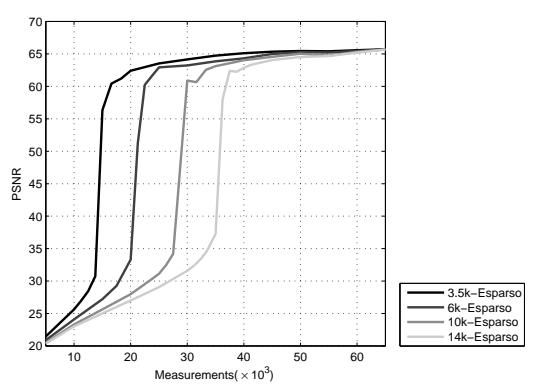

Fig. 2. Resultados para a aplicação do esquema DCT- $l_{1}-\mathrm{N}$ em versões esparsas da imagem Lena.

os obtidos para a compressão a partir da DCT linear (que seleciona $M$ coeficients do canto superior esquerdo da DCT), observamos que, enquanto CS depende apenas do fator de esparsidade $S$, a eficiência da compressão linear depende da distribuição dos coeficientes significativos no domínio DCT (veja Figura 3).

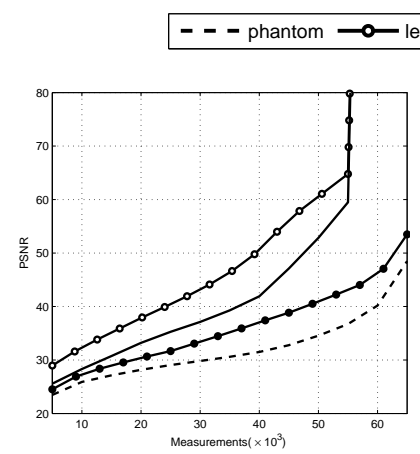

(a) Compressão Linear

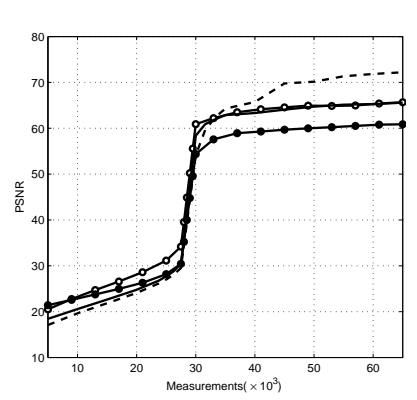

(b) Amostragem Compressiva
Fig. 3. A figura (a) apresenta resultados para a compressão linear quando uma esparsidade de $10 k$ é forçada às quatro imagens de teste e a figura (b) apresenta resultados para a estratégia DCT- $l_{1}$-L quando uma esparsidade de $10 k$ é forçada às quatro imagens de teste.

Quando utilizamos as imagens originais (sem forçar esparsidade), observamos que o domínio utilizado para a reconstrução apresenta forte influência no desempenho do método. A Figura 4 ilustra os resultados obtidos nas imagens Phantom e Lena comparando as estratégias descritas.

\section{QUANTIZAÇÃo}

Em geral, medidas não podem ser tomadas com precisão arbitrariamente grande e um erro de arredondamento é adicionado aos dados adquiridos. Este processo de quantização é muito importante para o estudo de aplicações de CS à aquisição de imagens, pois existe um grande interesse em comprimir o sinal. O tamanho do passo de quantização é extremamente relevante para a determinação da taxa de compressão que, por sua vez, é utilizada para avaliar a eficiência do método baseado no critério taxa-distorção.

Diferente do ruído Gaussiano, o erro de quantização é determinístico e depende do sinal original. Assim, uma contribuição significativa à teoria de CS consiste em verificar o desempenho na presença de erros de quantização e, em seguida, avaliar a técnica a partir do critério taxa-distorção.

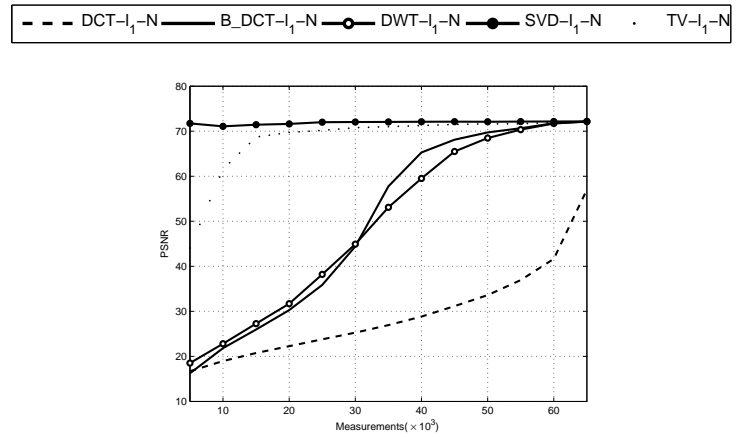

(a) Phantom

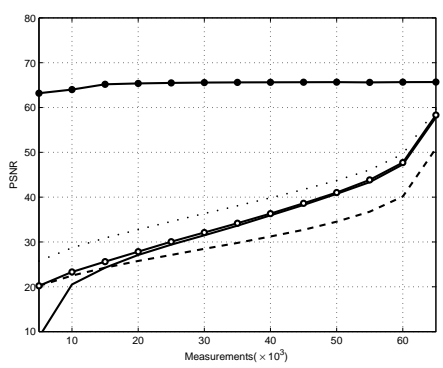

(b) Lena

Fig. 4. Resultados considerando erros de esparsidadesss.

\section{A. O Desempenho Taxa-Distorção}

Considerou-se a quantização escalar uniforme e foram realizados testes para diferentes passos de quantização. Selecionando para cada taxa de compressão o passo de quantização mais eficaz, é possível mostrar o desempenho taxa-distorção.

A taxa foi calculada com $(M / N) H_{y}$, onde $H_{y}$ é a entropia (em bits por pixel, estimada a partir do histograma de $y$ ) dos dados medidos $y$ após serem quantizados. O problema de valores quantizados não utilizados é solucionado assumindo-se que cada um ocorreu uma vez.

\section{RESUlTAdos ENVOLVENDO QUANTIZAÇÃO}

Na Figura 5, o desempenho taxa-distorção foi mostrado para todas as imagens de teste e estratégias de aquisição consideradas. Pode-se observar que o esquema de CS que realiza a minimização da norma $l_{1}$ no domínio Wavelet é significativamente menos eficiente que o padrão de compressão JPEG2000. No entanto, analisando os resultados obtidos para a estratégia SVD- $l_{1}-\mathrm{N}$ e para a imagem Phantom na estratégia TV-N, observa-se que existe espaço para o aprimoramento da técnica; em ambos os casos os desempenhos superam o do JPEG2000. Tanto o gradiente da imagem Phantom quanto a transformada SVD são altamente esparsos. Isto indica que, escolhendo-se uma representação que reforce esparsidade, é possível reduzir não apenas o número de medidas necessárias para reconstruir o sinal, como também o erro de aproximação.

É importante mencionar que, apesar da estratégia SVD- $l_{1}-\mathrm{N}$ representar um limite superior ao desempenho de CS, não se trata de um método praticável pois exige o conhecimento $a$ priori da DCT da imagem. A Figura 7 destaca este argumento contrastando a reconstrução da imagem Camera man tendo 


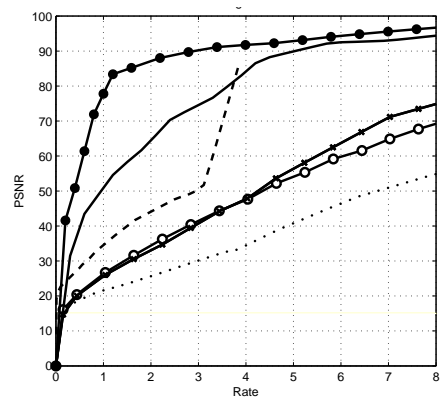

(a) Phantom

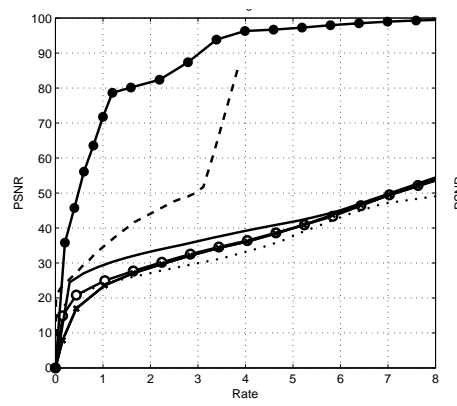

(b) Lena

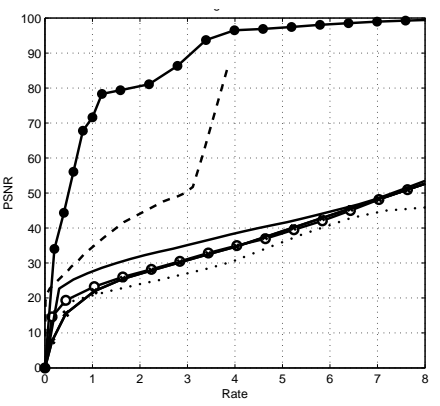

(c) Camera man

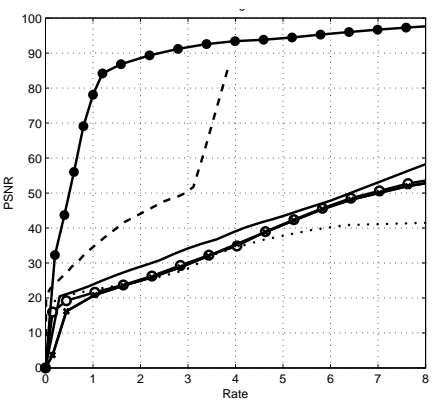

(d) Text

Fig. 5. Desemnpenho taxa-distorção para as estratégias de CS consideradas e o JPEG2000.

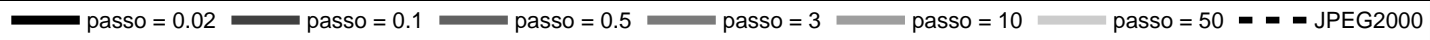

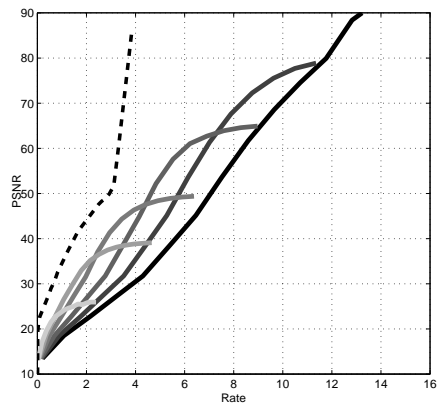

(a) Phantom

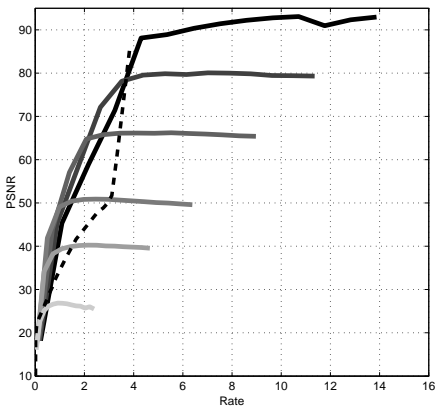

(e) Phantom

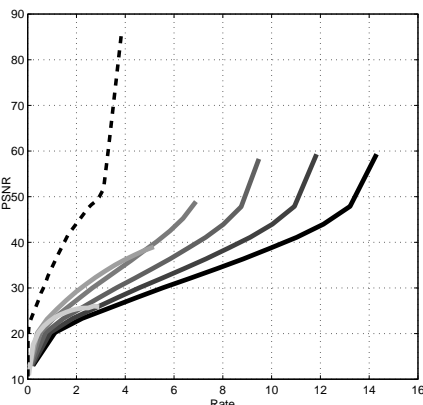

(b) Lena

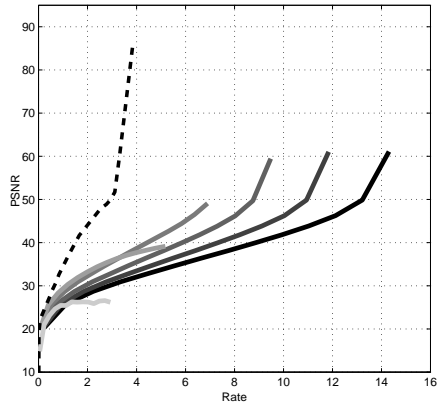

(f) Lena

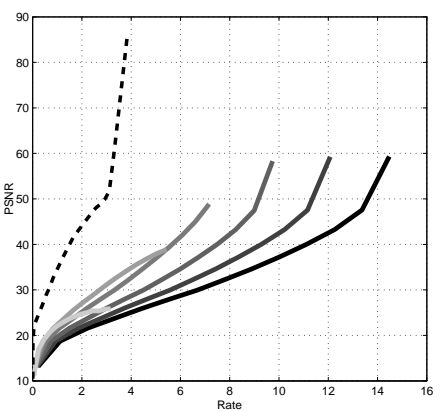

(c) Camera man

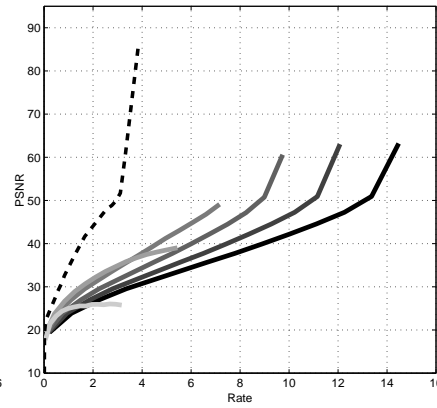

(g) Camera man

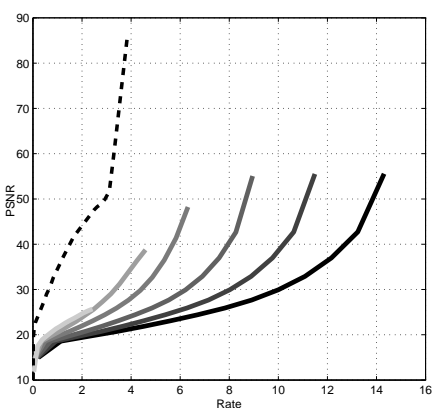

(d) Text

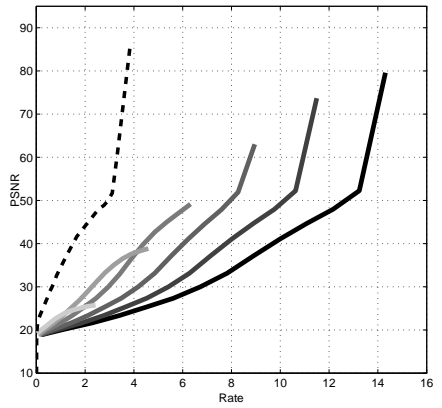

(h) Text

Fig. 6. Taxa $\times$ PSNR para passos de quantização variados: $(\mathrm{a}-\mathrm{d})$ estratégia DWT- $l_{1}-\mathrm{N}$ e (e-h) estratégia TV-N.

como base da representação esparsa a SVD de Camera man e de Lena.

Na Figura 6, o desempenho Taxa $\times$ PSNR foi mostrado para as estratégias DWT- $l_{1}-\mathrm{N}$ e TV-N para diversos passos de quantização. Pode ser constatado que, para uma determinada taxa de compressão, cada imagem e estratégia de reconstrução possui um passo de quantização ótimo que produz a maior PSNR. Se a imagem não for esparsa no domínio considerado, os resultados mostram que é mais eficiente tomar um grande número de medidas e compensar o aumento de taxa resultante aumentando o passo de quantização.

Também pode-se notar que, para uma PSNR fixa, o passo de quantização ideal é aproximadamente o mesmo em todos os cenários avaliados. Esta observação está altamente

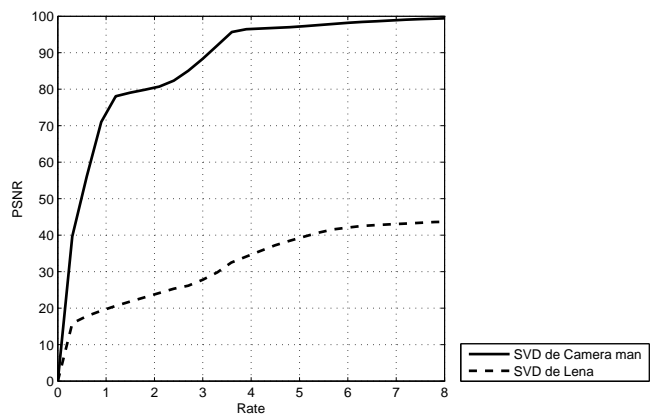

Fig. 7. Reconstrução da imagem Camera man a partir da estratégia SVD- $l_{1}-\mathrm{N}$. 


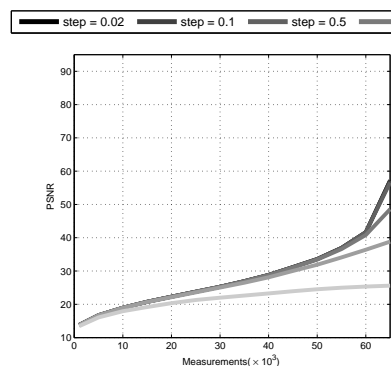

(a) Estratégia DCT- $l_{1}-\mathrm{N}$

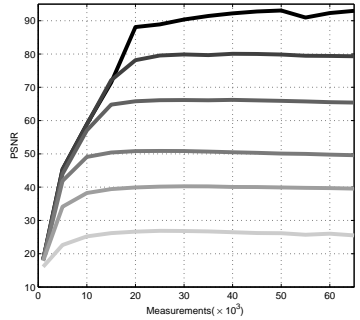

(c) Estratégia TV-N

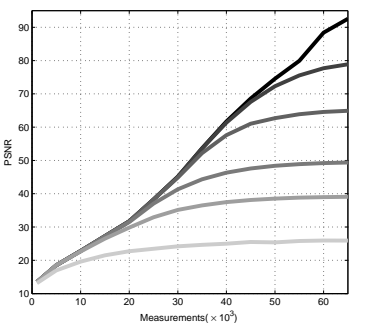

(b) Estratégia DWT- $l_{1}-\mathrm{N}$

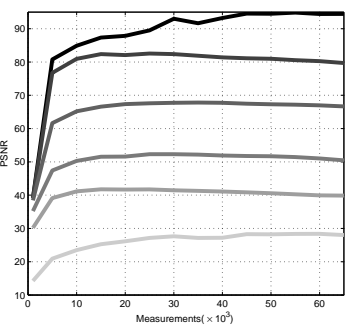

(d) Estratégia SVD- $l_{1}-\mathrm{N}$
Fig. 8. Número de Medidas $\times$ PSNR quando utilizados diferentes passos de quantização na aquisição CS da imagem Phantom.

relacionada ao resultado da Equação 4, que indica ser o erro de reconstrução da ordem do maior entre o erro de aproximação e de medições [3]. A PSNR determina a distorção aceitável e, portanto, os valores de $\epsilon_{q}$ e $\epsilon_{s}$. Enquanto $\epsilon_{q}$ apenas depende do passo de quantização, $\epsilon_{s}$ depende da esparsidade e, conseqüentemente, do número de medidas.

A mesma observação pode ser feita na Figura 8, que mostra os resultados em termos do Número de Medidas $\times$ PSNR. Para cada estratégia, o número de medidas determina o valor de $\epsilon_{s}$ e, além disso, todos os passos de quantização que fazem $\epsilon_{q}$ ser da ordem de $\epsilon_{s}$ (ou menor) resultam na mesma PSNR (veja a Equação 4). Assim, todas as curvas se sobrepõem até que o número de medidas seja grande o suficiente para que $\epsilon_{s}$ exceda $\epsilon_{q}$ (veja a Figura 8(b)). Na Figura 8(a), é notável que para passos de quantização menores do que 3 , as curvas se sobrepõem completamente. Isto ocorre pois, como os erros devido à esparsidade são muito grandes, a redução do passo de quantização é ineficaz para o aumento da PSNR. Em contrapartida, na Figura 8(d), onde a imagem é altamente esparsa no domínio considerado (SVD), $\epsilon_{s}$ tende a ser bem menor e, assim, este comportamento não é observado.

Uma lista completa de resultados e códigos estão disponiveis em www.impa.br/ aschulz/CS.

\section{Discussões E CONCLUSÕES}

Os resultados obtidos durante este estudo sugerem contextos nos quais aprimoramentos da técnica de CS poderiam resultar em melhores desempenhos referentes à avaliação taxadistorção .

Já foi enfatizado que a esparsidade exerce um papel fundamental na recuperação de sinais adquiridos via CS (e.g., SVD). Portanto, para tornar práticas as aplicações de CS em aquisição e compressão de imagens, devem ser investigados domínios que destaquem a esparsidade desses sinais, como bases biortogonais de Wavelets e modelos baseados em gradiente (e.g., $T V$ ).

Outro aspecto fundamental para o desenvolvimento desta teoria é o algoritmo de reconstrução. Apesar deste trabalho apenas avaliar a minimização da norma $l_{1}$ e da $T V$, existem algoritmos recentes que não apenas aceleram, mas também aprimoram a reconstrução [17].

Diferentes modelos de quantização também foram propostos como formas de melhorar o desempenho de CS [5]. Seria ainda interessante investigar matrizes de medidas alternativas as Noiselets.

Finalmente, é importante discutir a universalidade de CS. A grande maioria das publicações referenciadas enfatizam que uma das principais vantagens de CS consiste em ser um método não-adaptativo. Isto quer dizer que a codificação pode ser feita sem ser conhecida a distribuição dos coeficientes significativos na representação esparsa. De fato, as mesmas medidas aleatórias podem ser usadas para reconstruir qualquer sinal desde que exista um domínio no qual ele é esparso. No entanto, nos experimentos realizados, o melhor compromisso entre o número de medidas e o passo de quantização varia de acordo com a distribuição de esparsidade do sinal. Assim, a formulação de estratégias que atenuem este efeito é um tema para pesquisas adicionais.

\section{REFERÊNCIAS}

[1] Emmanuel Candès, Justin Romberg, and Terence Tao, "Robust uncertainty principles: Exact signal reconstruction from highly incomplete frequency information," IEEE Trans. on Information Theory, vol. 52, no. 2, February 2006.

[2] David L. Donoho, "Compressed sensing," IEEE Transactions on Information Theory, vol. 52, no. 4.

[3] Emmanuel Candès and Justin Romberg, "Encoding the $l_{p}$ ball from limited measurements," DCC, 2006.

[4] P. Boufounos and R. Baraniuk, "Quantization of sparse representations," DCC, 2007.

[5] W. Dai, H. V. Pham, and O. Milenkovic, "Distortion-Rate Functions for Quantized Compressive Sensing," ArXiv e-prints, Jan. 2009.

[6] A. Fletcher, S. Rangan, and V. Goyal, "On the rate-distortion performance of compressed sensing.," ICASSP, 2007.

[7] S. Sarvotham, D. Baron, and R. Baraniuk, "Measurements vs. bits: Compressed sensing meets information theory," Allerton Conf. on Communication, Control, and Computing, 2006.

[8] Adriana Schulz, Luiz Velho, and Eduardo A. B. da Silva, "On the empirical rate-distortion performance of compressive sensing," IEEE International Conference on Image Processing (ICIP), 2009.

[9] Emmanuel Candès and Michael Wakin, "An introduction to compressive sampling," IEEE Signal Processing Magazine, vol. 25, no. 2, March 2008.

[10] Emmanuel Candès and Justin Romberg, "Sparsity and incoherence in compressive sampling," Inverse Problems, vol. 23, no. 3, pp. 969-985, 2007.

[11] Emmanuel Candès and Terence Tao, "Decoding by linear programming," IEEE Trans. on Information Theory, vol. 51, no. 12, December 2005.

[12] Emmanuel Candès, "The restricted isometry property and its implications for compressed sensing," Compte Rendus de l'Academie des Sciences, Series, vol. 346, pp. 589-590, 2008.

[13] Emmanuel Candès, Justin Romberg, and Terence Tao, "Stable signal recovery from incomplete and inaccurate measurements," Communications on Pure and Applied Mathematics, vol. 59, no. 8, August 2006.

[14] Justin Romberg, "Imaging via compressive sampling," IEEE Signal Processing Magazine, vol. 25, no. 2, March 2008.

[15] Emmanuel Candès and Justin Romberg, "L1-magic," www.11magic.org.

[16] David L. Donoho, Arian Maleki, and Morteza Shahram, "Wavelab," http://www-stat.stanford.edu/ wavelab.

[17] E. Candès, M. Wakin, and S. Boyd, "Enhancing sparsity by reweighted $l_{1}$ minimization," J. Fourier Anal. Appl., 2007. 